\title{
Evaluación de recursos tecnológicos didácticos mediante e-rúbricas
}

\section{Assessment of Technological Teaching Resources through E-Rubrics}

\author{
Alba García-Barrera \\ Universidad a Distancia de Madrid. España. \\ alba.garcia@udima.es
}

\begin{abstract}
Resumen
Para introducir adecuada y efectivamente las TIC en el ámbito educativo se deben modificar los métodos y estrategias que estructuran el proceso de enseñanza-aprendizaje, ya que no pueden seguir respondiendo al enfoque expositivo tradicional. El profesorado debe ejercer un rol de facilitador del aprendizaje y el alumnado debe tomar un papel más activo. Pero también debe modificarse la evaluación y la elección que se realiza de las herramientas y materiales digitales a emplear en el aula. Ante esta necesidad, se invitó a 50 estudiantes de la Universidad a Distancia de Madrid a que, dentro de la asignatura de Magisterio denominada "Métodos, recursos y nuevas tecnologías para el aprendizaje", diseñaran una e-rúbrica que les sirviera para valorar la calidad y la adecuación de los recursos educativos digitales, y que al mismo tiempo les ofreciera la oportunidad de descubrir las posibilidades didácticas que brinda este tipo de herramienta como medio de evaluación.
\end{abstract}

\section{Palabras clave}

Evaluación, E-rúbrica, Recursos digitales, Formación del profesorado, Tecnologías de la Información y la Comunicación (TIC).

\begin{abstract}
To properly and effectively introduce ICT in education, the methods and strategies that structure the teaching-learning process must be changed, as they cannot continue responding to the traditional expositive approach. Teachers must play a role of learning facilitator and students must take a more active role. But the evaluation and selection that is made of digital tools and materials used in the classroom should also be modified. Given this need, 50 students from the Madrid Open University, who were studying the subject of Education entitled "Methods, resources and new technologies for learning", were invited to design an e-rubric which could serve them to assess the quality and the adequacy of digital educational resources, and at the same time it could offer them the opportunity to discover the educational possibilities offered by this type of tool as evaluation method.
\end{abstract}

\section{Key words}

Evaluation, E-rubric, Digital resources, Teacher training, Information and Communication Technologies (ICT).

\section{Introducción}

En el ámbito educativo español, la llegada de las TIC (Tecnologías de la Información y de la Comunicación) trajo consigo nuevas necesidades de formación para el profesorado, que se vio afectado por múltiples cambios. Entre ellos, la apuesta por un nuevo modelo pedagógico basado en un paradigma de enseñanza-aprendizaje centrado 
en el estudiante (Blanco, 2009; Serrano y Cebrián, 2014) y en el que el profesorado cambia su rol para convertirse en un acompañante cognitivo (Tedesco, 2011), pasando de ser un mero transmisor de conocimientos a un facilitador de los aprendizajes (Avello y López, 2015).

De esta forma, la función docente se acomoda a las nuevas demandas y potencialidades de su entorno, donde deja de tener sentido la perspectiva que considera al profesor como experto y principal fuente de información y respuestas (Resta, 2004; o.c. en Goig, 2014), ya que ahora toda esa información queda fácilmente accesible a través de internet. Así, en este nuevo modelo el alumnado deja de ser un mero receptor de contenidos para pasar a ejercer un papel activo en el proceso de enseñanza-aprendizaje, participando, colaborando, intercambiando experiencias con sus compañeros y construyendo sus propios conocimientos.

Sin embargo, pese a que la tecnología educativa encierra un enorme potencial tanto en este sentido como para el aprendizaje en sí, y que desde diversos organismos se ha reiterado la necesidad de utilizar las TIC y los recursos educativos abiertos en todos los contextos y niveles de aprendizaje (Comisión Europea, 2012), su integración en las prácticas educativas no está obteniendo los resultados esperados (Ertmer and OttenbreitLeftwich, 2010; Hixon and Buckenmeyer, 2009; Ramboll Management, 2006; o.c. Almerich, Suárez-Rodríguez, Belloch y Bo, 2011). De hecho, la introducción de las TIC en las aulas españolas ha sufrido múltiples reticencias entre los docentes, que a su vez han causado que en el currículo escolar, especialmente el de etapas obligatorias, aún no se haya establecido un lugar preferente para las TIC (Medina y Ballano, 2015).

Ello no significa que en nuestro país la presencia de las TIC en las aulas no haya tenido carácter progresivo, pero ciertamente aún no se ha producido un cambio notorio en las prácticas docentes (Sigalés, Mominó y Meneses, 2013) que permita desarrollar plenamente su integración didáctica.

Y es que con la introducción de las TIC el enfoque de dichas prácticas educativas debe replantearse, entendiendo que además de suponer una forma más atractiva y entretenida de abordar los contenidos, transforman el propio proceso y diseño de la enseñanza (Aguiar, Verdún, Silin, Capuano y Aristimuño, 2014). De hecho, se considera que un buen uso de las TIC implica acciones como la comprensión de conceptos y procedimientos tecnológicos y digitales, el desarrollo de estrategias didácticas de tipo constructivista, la integración en las tareas educativas de las experiencias y la formación previa del alumnado, y la comprensión de cómo las TIC pueden ayudar a superar las dificultades que presentan los estudiantes (Valverde, Garrido y Fernández, 2010).

La forma de evaluar todos estos procesos de forma que sean coherentes con la introducción y manejo de las TIC comienza a situarse como un importante foco en las investigaciones más actuales. Por ejemplo, un reciente estudio realizado por Aguiar, Verdún, Silin, Capuano y Aristimuño (2014), puso de relieve que esta cuestión se sitúa entre una de las preocupaciones que el profesorado manifiesta acerca de las TIC, considerando que las TIC pueden suponer una buena herramienta para evaluar de forma integral y personalizada a los estudiantes, pero que su implementación en las aulas debe 
llevar necesariamente aparejada un cambio en la forma de evaluar las prácticas educativas, tanto a nivel inicial como procesual y final.

\subsection{Evaluación mediante e-rúbricas}

En este contexto surge el auge de las rúbricas, entendidas como (Jonsson \& Svingby, 2007; Fernández March, 2010; Reddy \& Andrade, 2010; Cebrián y Monedero, 2014; Valverde y Ciudad, 2014; Marín-García y Santandreu-Mascarell, 2015) documentos que: a) describen los criterios que se van a seguir para evaluar un trabajo, tarea, producto o actividad; b) enumeran los distintos niveles de calidad de cada uno de dichos criterios con las características o parámetros que deben cumplir; y c) incluyen los puntos asignados a cada nivel de calidad y el peso correspondiente a cada criterio.

En relación a la fundamentación teórica y científica de las e-rúbricas, cabe destacar que recientemente Marín-García y Santandreu-Mascarell (2015) llevaron a cabo una revisión de 142 artículos científicos sobre estas herramientas, concluyendo que no existe suficiente evidencia científica que respalde su uso y diseño, ya que las investigaciones realizadas hasta el momento no poseen una metodología sólida y contrastada que indique en qué contexto y para qué pueden ser realmente útiles.

Sin embargo, las rúbricas han sido normalmente utilizadas como instrumentos y técnicas para la evaluación, y aunque en tanto metodología se han aplicado para diversos objetivos, niveles y modalidades de enseñanza, su uso más extendido ha resultado estar dirigido hacia la evaluación por competencias (Cebrián y Monedero, 2014).

Las TIC han contribuido a que las rúbricas, que tradicionalmente se usaban en papel, se desarrollen en un formato electrónico y más interactivo, constituyendo las rúbricas electrónicas o e-rúbricas (Cebrián, Martínez, Gallego y Raposo, 2011; Ciudad, 2011; Valverde y Ciudad, 2014). El diseño de las e-rúbricas se basa en la misma concepción pedagógica que las rúbricas tradicionales o "cuadradas" (aquellas que conforman tablas o rejillas) (Cebrián y Monedero, 2014), y actualmente existen múltiples recursos online que facilitan su creación y diseño (Vázquez-Cano, Martín-Monje y Fernández-Álvarez, 2014), entre los que se encuentra Rubistar (http://rubistar.4teachers.org), la aplicación que ha sido utilizada para llevar a cabo la presente experiencia.

No obstante, cabe destacar que, además de las reticencias ya mencionadas, referentes al diseño y la evaluación de los procesos tecnoeducativos, para la implementación de las erúbricas en las aulas existen también otras preocupaciones por parte del profesorado entre las que sin duda se encuentra la selección de los recursos y materiales digitales didácticos.

\subsection{Selección de recursos y materiales digitales}

Se puede afirmar que los recursos y materiales digitales están revolucionando las prácticas educativas. Sin embargo, exigen que el docente sepa identificar determinados factores en ellos y no los incorpore al aula sin que cumplan unos mínimos requisitos en cuanto a su calidad. De igual modo, para que una vez seleccionados tengan éxito en el 
aula, deberán siempre llevar aparejada la integración de las estrategias didácticas necesarias en su planificación y organización curricular (Rivero, 2013).

En este sentido, en primer lugar resulta primordial entender que lo importante a la hora de enseñar no es la tecnología en sí misma, sino la calidad de los procesos de enseñanza-aprendizaje que se producen y su coherencia con las actividades curriculares (Correa y Martínez, 2010). Y en segundo lugar, hay que tener presente que no todos los recursos o materiales tecnológicos sirven para enseñar cualquier disciplina ni son siempre válidos para cualquier espacio educativo (Rubia, Anguita, Jarrín y Ruiz, 2010).

Desde la inclusión de las TIC en las aulas, han sido numerosos los autores que han recomendado el análisis de ciertas dimensiones de los recursos digitales por medio de indicadores de credibilidad que permitan evaluar su calidad y utilidad educativa (Area, 2009; Area, Cepeda, González y Sanabria, 2010; Codina, 2000; Cooke, 2001; Fornas, 2003; Grassian, 1997; Johnson, 2012; Kapoun, 1998; Kovacs y Elkordy, 2000; MartínLaborda, 2005; Marzal, Calzada-Prado y Vianello, 2008; Nokelainen, 2006; Núñez, 2002; Pasch, 1997; PEI, 2008; Rivero, 2013). En general, las guías de evaluación que analizan estos factores surgieron en el ámbito de la biblioteconomía y en un principio se enfocaron a los recursos de información disponibles en internet. Algunos de los que mayor extrapolación han tenido al ámbito educativo son los siguientes criterios: autoridad, credenciales, objetividad, adecuación, claridad, consistencia, independencia, cobertura, usabilidad, navegabilidad, fiabilidad, imparcialidad, temporalidad, actualización, utilidad, credibilidad e interactividad.

En esta línea, estudios recientes, como el realizado por Flores y Rivero (2014), ponen de relieve la necesidad de generar un instrumento con criterios unificados que facilite el trabajo de análisis de este tipo de materiales para la enseñanza, ya que la selección de materiales y recursos digitales que efectúa el profesorado no suele responder a criterios personales previamente reflexionados y basados en la propia comprobación de su eficiencia didáctica, sino que más bien obedece a la facilidad de localización del recurso y a las recomendaciones que otros compañeros les transmiten o que encuentran en la red, lo que no asegura su calidad y pone en peligro su éxito en el aula. En este sentido conviene tener en cuenta que la simple disponibilidad de dichos recursos no garantiza que el profesorado lleve a cabo un cambio en sus métodos y estrategias de enseñanza para integrarlos en el currículum (Román, Cardemil y Carrasco, 2011).

La adecuada selección de los recursos digitales "incide en el éxito o fracaso escolar, al interactuar en contextos y situaciones reales de aprendizaje, favoreciendo la adquisición de conocimientos, desarrollo de habilidades y formación de valores (competencias del saber, saber-hacer, ser y saber-convivir), guiando al estudiante hacia procesos de autonomía e interacción con los demás" (Rivero, 2013, p. 43).

Por ello, y como paso previo a la enseñanza de estrategias pedagógicas que ayuden a integrar dichos medios a las didácticas específicas inherentes a cada disciplina, resulta fundamental dotar al profesorado de los conocimientos y competencias necesarias y suficientes para discernir el grado de adecuación y la calidad que presentan los materiales y recursos tecnológicos, aprendiendo así a evaluarlos antes de incorporarlos como medio o herramienta didáctica en sus clases. 
Con este objetivo se planteó la experiencia que se describe a continuación, proponiendo como una de las actividades de la asignatura de "Métodos, recursos y nuevas tecnologías para el aprendizaje" de las titulaciones de Magisterio en Educación Infantil y Magisterio en Educación Primaria de la Universidad a Distancia de Madrid el diseño de una e-rúbrica que permita a los futuros docentes evaluar la calidad de los materiales digitales que deseen emplear en el aula, fomentando así su competencia digital y facilitándoles unos criterios que les permitan seleccionar recursos tecnológicos fidedignos y adecuados para la práctica educativa.

\section{Metodología}

\subsection{Descripción de la experiencia}

Ante las necesidades detectadas y que ya han sido anteriormente descritas, se consideró urgente incorporar entre los contenidos de Magisterio una unidad didáctica enfocada en este sentido, de modo que dentro de la competencia digital a alcanzar al finalizar la formación inicial del profesorado, se adquiriesen los conocimientos y habilidades suficientes para poder seleccionar y evaluar la adecuación de los materiales y recursos tecnológicos que se deseen trabajar en el aula con los estudiantes.

La experiencia se desarrolló en el tercer curso de los Grados de Magisterio de Educación Infantil y Primaria de la Universidad a Distancia de Madrid (UDIMA), una institución de educación superior de carácter privado que imparte enseñanzas a distancia.

La tarea diseñada formaba parte de la Unidad 2 de la asignatura de carácter básico "Métodos, recursos y nuevas tecnologías para el aprendizaje", cuyos contenidos versaban sobre la evaluación de y mediante las TAC (Tecnologías para el Aprendizaje y el Conocimiento), y se esperaba que contribuyera a consolidar el aprendizaje adquirido a través del estudio de los distintos materiales didácticos proporcionados.

La actividad propuesta era de carácter voluntario y suponía solamente el 0,2\% de la calificación correspondiente a la evaluación continua de los alumnos. El porcentaje restante estaba distribuido como refleja el Gráfico 1, componiéndose del examen final presencial (60\% de la nota), dos controles (10\% de la nota), dos trabajos (20\%) y otras cuatro actividades que, junto a esta, suponían el 10\% restante de dicha calificación final. 


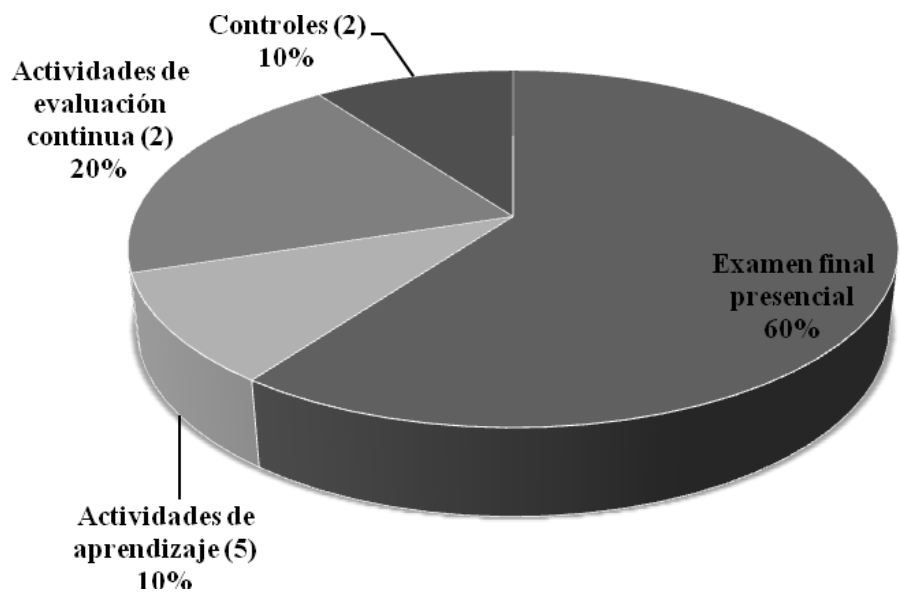

Gráfico 1. Distribución de puntuaciones en la evaluación continua.

\subsection{Población y muestra}

La población de estudio, compuesta por la totalidad de los estudiantes matriculados en dicha asignatura, que se encontraba situada en el primer semestre académico, ascendía a un total de 50 estudiantes.

Pese a la leve puntuación que llevaba asociada esta propuesta de actividad, fueron 40 los estudiantes que finalmente decidieron realizarla; esto es, el $80 \%$ de los alumnos matriculados en la asignatura.

De los participantes, 9 (22,5\%) fueron hombres y $31(77,5 \%)$ mujeres. Su edad oscilaba entre los 18 y los 55 años, siendo el grupo mayoritario el correspondiente al rango comprendido entre los 26 y los 30 años de edad, tal y como muestra el Gráfico 2.

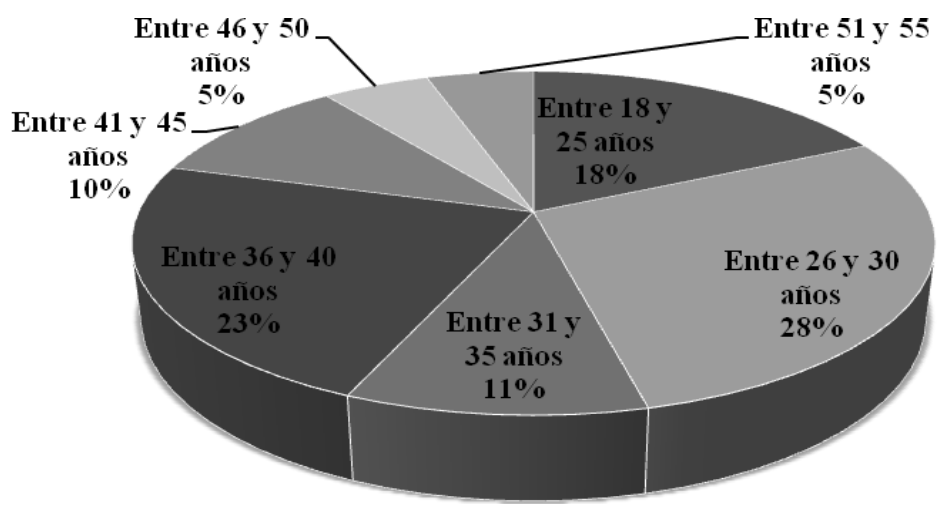

Gráfico 2. Edad de la muestra.

\subsection{Objetivos y procedimiento}

Entre los objetivos formativos de la actividad se encontraban los siguientes:

- Aprender a elaborar e-rúbricas utilizando la herramienta Rubistar.

- Experimentar una opción de evaluación mediante las TIC. 
- Reflexionar sobre la importancia de evaluar los recursos tecnológicos antes de utilizarlos en el aula.

- Identificar criterios para la adecuada selección de materiales y recursos educativos.

En general, se perseguía que los estudiantes integrasen los conocimientos adquiridos a través del estudio de la Unidad 2 de la asignatura con una experiencia práctica, que les sirviera tanto para tomar contacto con una de las herramientas disponibles para evaluar utilizando como medio las TIC, como para comprender la importancia de seleccionar adecuadamente los recursos que se utilizan en el aula evaluando también las propias TIC.

Para ello, se les invitó a tomar como referencia la información publicada en la página Educ.ar (http://www.educ.ar/recursos/ver?rec_id=93293), dependiente del Ministerio de Educación de Argentina.

A partir de estos indicadores, el estudiante tenía que elaborar una rúbrica con la herramienta Rubistar que incluyera aquellos criterios y requisitos que deberían cumplir los materiales y recursos educativos tecnológicos para resultar apropiados para el aprendizaje de los alumnos en el aula, examinando la información disponible en la red al respecto e incluyendo criterios propios, surgidos de su propia reflexión, conocimientos y experiencia.

\section{Resultados}

Los criterios que fueron empleados por la mayor parte de los estudiantes para parametrizar en la rúbrica la valoración de la calidad de los materiales didácticos digitales guardaron relación con los establecidos por autores como Downie (2011), Ur (1996) o Varela (2003) (o.c. en Vázquez-Cano, Martín-Monje y Fernández-Álvarez, 2014) y que pueden dividirse en las siguientes tres categorías:

- Criterios pedagógicos: adecuación del contenido y las actividades al nivel de desarrollo lingüístico y madurativo de los estudiantes; adaptación de la metodología utilizada; grado de dificultad, variedad y nivel de participación, tanto de estudiantes como de docentes.

- Criterios técnicos: funcionalidad de los materiales; integración de los medios y materiales empleados (auditivos, visuales, etc.).

- Criterios funcionales: posibilidad de integrar diversas destrezas y competencias en el proceso de enseñanza-aprendizaje.

De igual modo, los estudiantes tuvieron muy en cuenta los indicadores proporcionados por la página Educ.ar, completándolos en algunos casos con algunos aspectos relacionados con el proceso de enseñanza-aprendizaje, tales como el fomento de la motivación intrínseca o extrínseca del alumnado.

A través del siguiente enlace se puede consultar de forma online y en la propia plataforma de la herramienta Rubistar el resultado de una de las e-rúbricas diseñadas por uno de los estudiantes que cursaron la asignatura: http://rubistar.4teachers.org/index.php?screen=ShowRubric\&rubric_id=2480474\& 


\section{Discusión y conclusiones}

El enfoque implementativo que se ofrece en la presente propuesta de trabajo sobre las erúbricas difiere ampliamente de la perspectiva bajo la que habitualmente se emplean en el ámbito universitario, donde suelen utilizarse para la heteroevaluación (ya sea del profesor al alumno o de un alumno a otro alumno) o la autoevaluación de los estudiantes.

Así, la propia literatura que se ofreció a los alumnos matriculados en la asignatura de "Métodos, recursos y nuevas tecnologías para el aprendizaje" se centraba en dicha cuestión, dándoles a conocer esta herramienta como recurso para facilitar los procesos de evaluación en el aula. Por tanto, se consideró relevante que los estudiantes tuvieran la oportunidad de probar la herramienta de un modo distinto, cambiando en este caso no solo el destinatario de dicha evaluación (que dejaba de ser un estudiante), sino también el formato de la misma, que se convertía en un registro de observación en línea que daba como resultado el nivel de adecuación del material digital analizado.

En este sentido se puede afirmar que la experiencia resulta innovadora, en tanto no existe apenas literatura científica específica sobre el uso de las e-rúbricas para evaluar materiales digitales (Vázquez-Cano, Martín-Monje y Fernández-Álvarez, 2014).

Solamente se han encontrado experiencias similares enfocadas al análisis de materiales para didácticas específicas, como el estudio realizado por Vázquez-Cano, Martín-Monje y Fernández-Álvarez (2014), que muestra que el empleo de e-rúbricas para evaluar materiales enfocados a la enseñanza de lenguas optimiza la observación de la funcionalidad y utilidad del formato y conectividad de los elementos audiovisuales de este tipo de materiales digitales en red.

En cuanto a los resultados del uso general de las e-rúbricas en el ámbito educativo, los resultados obtenidos en la investigación llevada a cabo por Serrano y Cebrián (2014) las sitúan como una herramienta de evaluación con un alto grado de usabilidad y de satisfacción por parte de los estudiantes que las utilizan en su formación académica.

La experiencia desarrollada en este artículo presenta, además, el valor añadido de analizar el aprendizaje sobre evaluación también desde el punto de vista del futuro evaluador (Bay \& Kotaman, 2011; Cebrián, 2009; Cebrián, Martínez, Gallego y Raposo, 2011; López Pastor, Manrique y Vallés, 2011; Manrique et al., 2010; Martínez, Tellado y Raposo, 2013; Panadero, Alonso-Tapia \& Reche, 2013; o.c. en Gallego y Raposo-Rivas, 2014), por lo que en este sentido podría afirmarse que su implementación en la formación de los futuros maestros resultaría enriquecedora.

En cuanto a la evaluación de los materiales didácticos digitales, es importante recordar que no debe limitarse únicamente a examinar las características de los mismos, sino que para que realmente sean efectivos en el proceso didáctico, además debe tenerse en cuenta tanto el contexto en el que van a utilizarse como su influencia sobre el aprendizaje de los estudiantes (Aguilar, Ayala, Lugo y Zarco, 2014). 
Por último, cabe destacar que la introducción de esta experiencia en la formación inicial del profesorado dota a los estudiantes de Magisterio de aquellos conocimientos, medios y habilidades que les preparan no solamente para desarrollar la competencia digital de sus futuros alumnos, sino también a reforzar la propia, lo que puede ayudar a fomentar un mayor aprovechamiento de las oportunidades que ofrecen las TIC en el aula (Hoyos, 2013).

Presentación del artículo: 5 de octubre de 2016

Fecha de aprobación: 2 de abril de 2016

Fecha de publicación: 30 de abril de 2016

García-Barrera, A. (2016). Evaluación de recursos didácticos tecnológicos mediante erúbricas. RED. Revista de Educación a Distancia. 49(13). Consultado el (dd/mm/aaaa) en http://www.um.es/ead/red/49

\section{Referencias}

Aguiar, D., Verdún, N., Silin, I., Capuano, A. y Aristimuño, F. (2014). Las TIC en la educación media: ¿una herramienta más o nuevo contexto de aprendizaje? Análisis de las representaciones de docentes y directivos sobre el Programa Conectar Igualdad en tres provincias de la Patagonia Argentina. Magistro, 8(15), 19-58.

Aguilar, I. Ayala, J., Lugo, O. y Zarco, A. (2014). Análisis de criterios de evaluación para la calidad de los materiales didácticos digitales. Revista CTS, 25(9), 73-89. Recuperado de http://www.revistacts.net/files/Volumen\%209\%20\%20N\%C3\%BAmero\%2025/FINAL/AguilarFINAL.pdf

Almerich, G., Suárez-Rodríguez, J.M., Belloch, C. y Bo, R.M. (2011). Las necesidades formativas del profesorado en TIC: perfiles formativos y elementos de complejidad. RELIEVE, 17(2). Recuperado de http://www.uv.es/RELIEVE/v17n2/RELIEVEv17n2_1.htm

Area, M. (2009). Introducción a la Tecnología Educativa. Tenerife: Universidad de La Laguna. Recuperado de http://manarea.webs.ull.es/wpcontent/uploads/2010/06/ebookte.pdf

Area, M., Cepeda, O., González, D. y Sanabria, A. (2011). Un análisis de las actividades didácticas con TIC en aulas de educación secundaria. Pixel-Bit. Revista de Medios y Educación, 38(1), 187-199. Recuperado de http://www.redalyc.org/articulo.oa?id=36816200015

Avello, R. y López, R. (2015). Alfabetización digital de los docentes de las escuelas de hotelería y turismo cubanas. Experiencias en su implementación. RUSC. Universities and Knowledge Society Journal, 12(3), 3-15. doi: http://dx.doi.org/10.7238/rusc.v12i3.1994

Blanco, A. (2009). Desarrollo y evaluación de competencias en Educación Superior. Madrid: Narcea. 
Cebrián, M., Martínez, M.E., Gallego, M.J. y Raposo, M. (2011). La e-rúbrica para la evaluación: una experiencia de colaboración inter-universitaria en materia TIC. En II Congreso Internacional Usos y Buenas Prácticas TIC. Málaga. Recuperado de http://erubrica.uma.es/wp-content/uploads/2011/06/Comunicaci\%C3\%B3n.pdf

Cebrián, M. y Monedero, J.J. (2014). Evolución en el diseño y funcionalidad de las rúbricas: desde las rúbricas "cuadradas" a las erúbricas federadas. REDU: Revista de Docencia Universitaria, 12(1), 81-98. Recuperado de http://redu.net/redu/index.php/REDU/article/view/744/pdf

Ciudad, A. (2011). Design of the Accounting Course Focusing On the Development of Competences. Journal of International Education Research, 7(5),15-22. Recuperado de http://journals.cluteonline.com/index.php/JIER/article/view/6112/6190

Codina, L. (2000). Parámetros e indicadores de calidad para la evaluación de recursos digitales. En 7as Jornadas Españolas de Documentación (pp. 135-144). Bilbao: Universidad del País Vasco.

Comisión Europea (2012). Replantear la educación. Bruselas, 20 de noviembre. Recuperado de http://europa.eu/rapid/press-release_IP-12-1233_es.htm

Cooke, A. (2001). A guide to finding quality information on the Internet: selection and evaluation strategies. 2nd ed. London: Library Association.

Correa, J.M. y Martínez, A. (2010). ¿Qué hacen las escuelas innovadoras con la tecnología? Las TIC al servicio de la escuela y la comunidad en el colegio Amara Berri. T Teoría de la educación: educación y cultura en la sociedad de la información, 11(3), 230-261. Recuperado de http://campus.usal.es/ revistas_trabajo/index.php/revistatesi/article/view/5841/5867

Fernández March, A. (2010). La evaluación orientada al aprendizaje en un modelo de formación por competencias en la educación universitaria. REDU: Revista de Docencia Universitaria, 8(1), 11-34. Recuperado de http://redu.net/redu/files/journals/1/articles/144/public/144-130-2-PB.pdf

Flores, H.C. y Rivero, P. (2014). ¿Cómo selecciona el profesorado recursos digitales para enseñar el Historia? CLIO. History and History teaching, 40. Recuperado de http://clio.rediris.es/n40/articulos/FloresRivero2014.pdf

Fornas, R. (2003). Criterios para evaluar la calidad y fiabilidad de los contenidos en internet. Revista Española de Documentación Científica, 26(1), 75-80. Recuperado de http://redc.revistas.csic.es/index.php/redc/article/viewFile/22

Gallego, M.J. y Raposo-Rivas, M. (2014). Compromiso del estudiante y percepción del proceso evaluador basado en rúbricas. REDU: Revista de Docencia Universitaria, 12(1), 197-215. Recuperado de http://redu.net/redu/index.php/REDU/article/view/745/pdf 
Goig, R.M. (2014). La competencia pedagógica-digital del profesorado, factor clave en el proceso de cambio e innovación de la sociedad 2.0 a la sociedad 3.0. En R.M. Goig (Dir.), Formación del profesorado en la sociedad digital. Investigación, innovación y recursos didácticos, (pp. 9-28). Madrid: UNED.

Grassian, E. (1997). Thinking Critically about World Wide Web Resources. California: UCLA College Library. Recuperado de http://www.mscare.org/?page=Article0002

Hoyos, L. (2013). Competencia digital docente. Marpadal Interactive Media, S.L.

Johnson, S. (Ed.) (2012). Key issues for e-resource collection development: a guide for libraries. International Federation of Library Associations and Institutions (IFLA). Recuperado de http://www.ifla.org/files/assets/acquisition-collectiondevelopment/publications

Jonsson, A., \& Svingby, G. (2007). The use of scoring rubrics: Reliability, validity and educational consequences. Educational Research Review, 2(2), 130-144. doi: http://dx.doi.org/10.1016/j.edurev.2007.05.002

Kapoun, J. (1998). Teaching undergrads WEB evaluation. A guide for library instruction. College and Research Libraries News. Recuperado de http://library.cooper.edu/instruct/website_eval.pdf

Kovacs, D.K. y Elkordy, A. (2000). Collection development in cyberspace: building an electronic library collection. Library Hi Tech, 18(4). Recuperado de http://www.highschoollibrarian.com/SJSU/266/readings/kovacs_collection_cyberspa ce.doc

Marín-García, J.A. y Santandreu-Mascarell, C. (2015). ¿Qué sabemos sobre el uso de rúbricas en la evaluación de asignaturas universitarias? Intangible Capital, 11(1), 118-145. doi: http://dx.doi.org/10.3926/ic.538

Martín-Laborda, R. (2005). Las nuevas tecnologías en la educación. Madrid: Fundación AUNA. Recuperado de http://www.telecentros.info/pdfs/05_06_05_tec_edu.pdf

Marzal, M.A., Calzada-Prado, J. y Vianello, M. (2008). Criterios para la evaluación de la usabilidad de los recursos educativos virtuales: un análisis desde la alfabetización en información. Information Research, 13(4). Recuperado de http://www.informationr.net/ir/13-4/paper387.html

Medina, A. y Ballano, S. (2015). Retos y problemáticas de la introducción de la educación mediática en los centros de Secundaria. Revista de Educación, 369(3), 135-158.

Nokelainen, P. (2006). An empirical assessment of pedagogical usability criteria for digital learning material with elementary school students. Educational Technology \& Society, 9(2), 178-197. Recuperado de http://ifets.info/journals/9_2/15.pdf 
Núñez, M. (2002). Criterios para la evaluación de la calidad de las fuentes de información sobre salud en Internet. ACIMED, 10(5). Recuperado de http://bvs.sld.cu/revista/aci/vol10_5_02/aci05502.html

Pasch, G. (1997). Generación de productos de calidad en Internet. En $V$ Foro Latinoamericano de Información, LatinBase '97. Recuperado de https://www.ischool.utexas.edu/ gpasch/cal.doc.html

PEI (2008). Evaluation and Selection of Learning Resources: A Guide. Canadá: Prince Edward Island. Recuperado de http://www.gov.pe.ca/photos/original/ed_ESLR_08.pdf

Reddy, Y.M., \& Andrade, H. (2010). A review of rubric use in higher education. Assessment \& Evaluation in Higher Education, 35(4), 435-448. doi: http://dx.doi.org/10.1080/02602930902862859

Rivero, I. (2013). Criterios para seleccionar tecnologías educativas y estrategias didácticas en el Colegio Guillermo León Valencia. Educación y ciencia, 16, 37-52. Recuperado http://revistas.uptc.edu.co/revistas/index.php/educacion_y_ciencia/article/view/3239

Román, M., Cardemil, C. y Carrasco, Á. (2011). Enfoque y metodología para evaluar la calidad del proceso pedagógico que incorpora TIC en el aula. Revista Iberoamericana de Evaluación en Educación, 4(2), 8-35. Recuperado de http://www.rinace.net/riee/numeros/vol4-num2/art1.pdf

Rubia, B., Anguita, R., Jarrín, I. y Ruiz, I. (2010). Teoría de la educación: educación y cultura en la sociedad de la información, 11(3), 96-120. Recuperado de http://campus.usal.es/ revistas_trabajo/index.php/revistatesi/article/view/5790/5820

Serrano, J. y Cebrián, D. (2014). Usabilidad y satisfacción de la e-rúbrica. REDU: Revista de Docencia Universitaria, 12(1), 177-195. Recuperado de http://redu.net/redu/index.php/REDU/article/view/775/pdf

Sigalés, C., Mominó, J.M. y Meneses, J. (2013). TIC e innovación en la educación escolar española. Estado y perspectivas. En A. Sacristán, (Comp.), Sociedad del conocimiento, tecnología y educación, (pp. 305-318). Madrid: Morata.

Tedesco, J.C. (2011). Los desafíos de la educación básica en el siglo XXI. Revista Iberoamericana de Educación, 55, 31-47. Recuperado de http://www.rieoei.org/rie55a01.pdf

Valverde, J. y Ciudad, A. (2014). El uso de e-rúbricas para la evaluación de competencias en estudiantes universitarios. Estudio sobre fiabilidad del instrumento. REDU: Revista de Docencia Universitaria, 12(1), 49-79. Recuperado de http://redu.net/redu/index.php/REDU/article/view/724/pdf

Valverde, J., Garrido, M.C. y Fernández, R. (2010). Enseñar y aprender con tecnologías: Un modelo teórico para las buenas prácticas con TIC. Teoría de la 
educación: educación y cultura en la sociedad de la información, 11(3), 203-229. Recuperado

http://campus.usal.es/ revistas_trabajo/index.php/revistatesi/article/view/5840/5866

Vázquez-Cano, E., Martín-Monje, E. y Fernández-Álvarez, M. (2014). El rol de las erúbricas en la evaluación de materiales digitales para la enseñanza de lenguas en entornos virtuales de aprendizaje. REDU: Revista de Docencia Universitaria, 12(1), 135-157. Recuperado de http://red-u.net/redu/index.php/REDU/article/view/756/pdf 